\title{
COMPARACIÓN ENTRE LA CORRECCIÓN CON MALLAS SINTÉTICAS VÍA VAGINAL DEL PROLAPSO GENITAL VERSUS TÉCNICAS VAGINALES TRADICIONALES. CLÍNICA UNIVERSITARIA BOLIVARIANA, MEDELLÍN, COLOMBIA 2006-2007*
}

\section{Vaginal synthetic mesh management for pelvic organ prolapse compared to traditional techniques used at the Clínica Universitaria Bolivariana, Medellín, Colombia, 2006-2007}

Luis G. Echavarría-Restrepo, M.D., MSc**, Juan A. Londoño-Montoya, M.D.***, Luis F. Trujillo-Gallego, M.D.****, Liliana P. Montoya-Vélez, M.D., MSc*****

Recibido: octubre 16/07 - Aceptado: junio 9/08

\section{RESUMEN}

Objetivo: comparar las técnicas de corrección de fallas del sostén del piso pélvico que utilizan mallas, con las técnicas tradicionales.

Métodos: cohortes de pacientes intervenidas en la Clínica Universitaria Bolivariana, Medellín, Colombia. Una cohorte utilizó mallas sintéticas y la otra técnica tradicional. Se evaluaron las complicaciones tempranas y tardías, y la recurrencia de defectos de la pared anterior y recidiva del prolapso de cúpula.

* Trabajo ganador del premio en la categoría Trabajos Libres Ginecología, presentado en el V Congreso Mundial de Perinatologia y XXVI Congreso Colombiano de Obstetricia y Ginecología, realizado en Cartagena del 28 al 31 de mayo del 2008.

** Profesor titular y coordinador del posgrado de ginecología y obstetricia UPB, Magíster en Salud Pública y Epidemiología. Carrera 72A No. 78B 50. Clínica Universitaria Bolivariana. Medellín, Colombia. Correo electrónico: echavarrialuis@yahoo.es

** Especialista en ginecología y obstetricia. Área de ginecología y obstetricia UPB.

*** Profesor asociado área de ginecología y obstetricia UPB.

*****Magíster en Epidemiología. Profesora asistente Maestría en Epidemiología CES.
La evaluación la realizó un ginecólogo que no intervino las pacientes y desconocía la técnica empleada. El seguimiento fue hecho a los 6 y 12 meses de la cirugía. Se compararon los dos grupos mediante el riesgo relativo y los intervalos de confianza. Se solicitó consentimiento informado.

Resultados: no hubo recaídas en las cirugías del compartimiento posterior y se observó tendencia a menor falla en las cirugías de la región apical usando mallas. A los seis meses de seguimiento se encontró un riesgo relativo $=0,26$, IC 95\% $=0,10-0,69$, $\mathrm{p}=0,001$ a favor de la técnica con malla en la reparación del compartimiento anterior y al año se confirmó esta técnica como protectora de la recaída $(\mathrm{RR}=0,32, \mathrm{IC} 95 \%=0,12-0,81, \mathrm{p}=0,004)$.

Discusión: el reto del cirujano pélvico es el control del compartimiento anterior. Los defectos paravaginales casi nunca son contemplados en las técnicas de corrección tradicional, estos son objeto primordial en el manejo integral con mallas para disminuir la 
recurrencia. En la cohorte intervenida con mallas se busca la neoformación de tejidos, ubicando el material sintético en los reparos anatómicos para rescatar el mecanismo funcional de la continencia. La revisión sistemática de Cochrane (2007) coincide con los resultados, privilegiando la corrección del compartimiento anterior con material sintético y confirmando la menor tasa de recaídas.

Palabras clave: prolapso vaginal, cistocele, prolapso órganos pélvicos, fijación sacroespinosa, material sintético.

\section{SUMMARY}

Objective: evaluating the recurrence of anterior wall and pelvic apical prolapse by comparing techniques for correcting pelvic failure via integral management using synthetic mesh compared to traditional ones.

Methods: two cohort groups were evaluated at the Clínica Universitaria Bolivariana in Medellín, Colombia; the first group was treated with surgical vaginal synthetic mesh and traditional techniques were used with the second one. Two evaluations were carried out at six-month intervals following the surgery. Follow-up involved classifying the degree of recurrence and early or late complications. The gynaecologist carrying out the follow-up did not know the patients and did not have any information about the cohorts. Informed consent had been previously signed by each patient.

Results: the most commonly recurring prolapse happened in the anterior compartment but occurred less frequently when vaginal synthetic mesh was used. No prolapses recurred in the posterior compartment in this cohort and a less frequent apical failure rate was also detected in this group. The techniques used in integral management protected against surgical failure. Six-month follow-up relative risk was $0.26(0.10-0.6995 \% \mathrm{CI} ; \mathrm{p}=0.001)$. One-year follow-up confirmed that this technique did protect against anterior compartment failure $(\mathrm{RR}=0.32 ; 0.12-0.81$ 95\% $\mathrm{CI} ; \mathrm{p}=0.004)$.

Discussion: the surgical techniques used in integral management did protect against surgical failure. The greatest challenge for a pelvic surgeon lies in controlling the anterior compartment because of the different kinds of pelvic failure. Para-vaginal and posterior defects are not usually fixed during abdominal or traditional surgery, although they do become the target during integral management. The synthetic mesh used on the exposed cohort was placed in anatomical sites, aimed at producing tissue neo-formation and thereby rescuing the functional continence mechanism. A systematic review of Cochrane data (2007) supported this paper's results, reinforcing integral anterior compartment management and confirming that synthetic material use leads to a lower rate of recurrence.

Key words: pelvic organ prolapse, mesh, synthetic material, integral management.

\section{INTRODUCCIÓN}

El prolapso genital femenino es un problema de salud pública, su frecuencia aumenta con la edad y afecta a la mitad de las mujeres mayores de 50 años, con una prevalencia del 30\%-50\%. ${ }^{1}$ Se ha estimado que una mujer durante su vida tiene un riesgo de ser llevada a cirugía por prolapso en un $11,1 \% .{ }^{1} \mathrm{La}$ corrección quirúrgica se acompaña de frecuentes recaídas por fallas en la pared o compartimiento anterior (cistocele) aproximadamente en un 30\% de las pacientes, y hasta un 3\% a 5\% de recidiva del prolapso de cúpula. ${ }^{2,3}$ Uno de los mayores problemas a que se enfrenta el cirujano pélvico es la elección de la técnica quirúrgica, ${ }^{4}$ ya que el origen del defecto es básicamente la falla de la capacidad de los tejidos de mantener el sostén por trastornos del colágeno, ${ }^{5}$ hipoestrogenismo ${ }^{6}$ o elongación y debilidad marcada, ${ }^{7}$ secundarios a factores de riesgo bien definidos por la literatura mundial ${ }^{4,8}$ y que son estos mismos tejidos y ligamentos los que se usan en la mayoría de técnicas convencionales para corregir el defecto, especialmente cuando se corrige el compartimiento anterior (cistocele). ${ }^{9}$ En la corrección de defectos del componente apical, principalmente el prolapso de la cúpula vaginal y la hernia pélvica, se 
utilizan otras estructuras corporales para la fijación que exigen intervenciones mayores y gran destreza quirúrgica como son la colposacropexia por vía abdominal o la fijación a ligamento sacroespinoso por vía vaginal. ${ }^{10,11}$

Como alternativa surge el abordaje quirúrgico con material sintético. ${ }^{12}$ Se trata de un material de polipropileno macroporoso que permite ser colonizado por tejido vivo, y por último remplazado por este, formando neoligamentos que logran el sostén del piso pélvico y restablecen la anatomía normal del mismo. ${ }^{13,14}$ Algunas publicaciones refieren con estas técnicas tasas menores de recurrencia y recidivas de los defectos originales. ${ }^{15}$ Hay poca información acerca de la evaluación óptima de estas técnicas mediante experimentos clínicos controlados. En Colombia no está popularizada la técnica con mallas para el defecto pélvico y solo a finales del año 2005 se realizaron las primeras cirugías.

El objetivo del estudio fue comparar las técnicas tradicionales de corrección de fallas del sostén del piso pélvico, con aquellas que utilizan mallas mediante la evaluación de la recurrencia de defectos de la pared anterior y recidiva del prolapso de cúpula.

\section{MATERIALES Y MÉTODOS}

Se realizó un estudio prospectivo de cohortes. Una cohorte recibió técnicas quirúrgicas vaginales colocando mallas sintéticas y la otra cohorte fue sometida a las técnicas tradicionales vaginales para el manejo estándar de la corrección del prolapso genital.

Población y lugar: pacientes con prolapso genital atendidas en la Clínica Universitaria Bolivariana, hospital general de atención que atiende población perteneciente al régimen contributivo, entre enero del 2006 y enero del 2007.

Criterios de inclusión: pacientes que al utilizar la clasificación de la ACOG tenían prolapso igual o mayor de II y fueran sintomáticas. Se excluyeron aquellas pacientes que no aceptaron participar en el estudio luego de cumplir los criterios de ingreso.

Tamaño muestral y muestreo: se calculó el tama- ño de la muestra con el programa Epi Info versión 6 con un nivel de confianza del 95\%, un poder del $80 \%$, una incidencia de recurrencia en la técnica tradicional del 30\% y un riesgo relativo de 2,2, encontrando que el número de pacientes necesarias para el estudio es de 30 en cada uno de los grupos. Muestreo secuencial por conveniencia.

Procedimiento: las pacientes fueron seleccionadas a partir de la consulta de piso pélvico, una vez se verificaron los criterios de selección, se explicó la intervención y se dio para firma el consentimiento informado.

La cohorte con la aplicación de mallas fue intervenida por dos cirujanos pélvicos de reconocida trayectoria en el tema. Esto con el fin de evitar que cirujanos en plena curva de entrenamiento de la técnica quirúrgica distorsionaran los resultados finales ocasionando un sesgo de resultado. Las mallas utilizadas para la corrección quirúrgica fueron aquellas constituidas por materiales de polipropileno macroporoso monofilamento. El otro grupo fue intervenido por los especialistas del servicio de acuerdo con las técnicas tradicionales.

Se practicaron dos evaluaciones con intervalo de seis meses, a partir de la cirugía. El primer seguimiento consistió en clasificar el grado de relajación. Existen básicamente tres clasificaciones para el prolapso genital: la clásica que incluía solo III grados para el prolapso y las propuestas por el Colegio Americano y la Sociedad Internacional de Continencia que es la más objetiva, que contemplan IV grados de prolapso, no obstante, hasta el momento no existe un consenso generalizado que acepte una de ellas especialmente. Esto dificulta no solo la comunicación científica, sino también la comparación de los resultados terapéuticos. Nosotros aplicamos la clasificación del Colegio Americano de Ginecología y Obstetricia (ACOG), grado 1: descenso del punto de referencia de la lesión anatómica, entre la posición normal y la espina isquiática; grado 2: el punto de referencia está entre la espina isquiática y el himen; grado 3: el descenso llega hasta el himen; y grado 4: el 
descenso va más allá del himen. Se recolectaron las variables pertinentes a este tiempo de evaluación (recaídas, recurrencias, y complicaciones tardías). Igual procedimiento se aplicó en la segunda evaluación al año de la intervención. El evaluador estuvo enmascarado sobre el tipo de cirugía a la que fue intervenida la paciente, ya que no tuvo acceso a la historia clínica y ninguna de las dos técnicas utilizadas presenta una cicatriz quirúrgica que identifique con facilidad la técnica empleada.

Los eventos evaluados como complicaciones tempranas o posquirúrgicas fueron: sangrado posoperatorio, infección urinaria, cuadro febril, lesiones de tipo nervioso y tromboflebitis superficial. Los eventos por evaluar como complicaciones tardías fueron: absceso de cúpula vaginal, dispareunia, disquecia, incontinencia urinaria y disuria.

Se identificaron las variables a medir: clasificación de la severidad del prolapso en estadios II III y IV. Los defectos se clasificaron en falla del componente apical que incluyó la hernia pélvica y el prolapso de cúpula con o sin celes relacionados y falla de la pared anterior. Las técnicas quirúrgicas empleadas fueron la corrección de celes en los prolapsos grado II, la histerectomía vaginal y plastias para el grado III; cuando se tenía hernia pélvica o prolapso de cúpula se adicionó la fijación al ligamento sacroespinoso, en el grupo de malla esta se adicionó a la técnica en el momento de la corrección. Se incluyeron: complicaciones intraoperatorias tempranas y tardías, y porcentaje de recaídas por cada uno de los compartimientos hasta un año de intervenidas.

Se hizo análisis descriptivo de cada cohorte, se calcularon medidas propias de estos estudios, incidencias en expuestos y no expuestos, riesgo relativo, su respectivo intervalo de confianza y el valor de $\mathrm{p}$.

Los aspectos éticos se ajustan a la Declaración de Helsinki y la Resolución 8430 de 1993 del Ministerio de Salud, se clasifica como investigación con riesgo mínimo, ya que se hizo seguimiento a dos cohortes de pacientes ya intervenidas, se les solicitó consentimiento informado para la participación en el estudio en un formato diseñado para ello. La ejecución está avalada por el comité de ética de las universidades CES y UPB.

\section{RESULTADOS}

En el periodo de estudio se incluyeron 31 pacientes en el grupo con malla y 30 con la técnica tradicional. El seguimiento fue completo a los seis meses, al año fueron evaluadas 21 pacientes con malla y 25 con manejo tradicional.

En la tabla 1 se presentan las características sociodemográficas de cada cohorte; sin diferencias estadísticamente significativas, que confirman que la técnica quirúrgica es la variable de mayor peso, responsable de los resultados encontrados al evaluar el seguimiento.

La suma de los defectos del componente apical de la cohorte con malla fueron del $81 \%$, y en el grupo tradicional de 87\%, $(p=0,772)$. El diagnóstico de falla del compartimiento anterior es de $17 \%$ en la cohorte con malla y del 13\% en la cohorte de manejo tradicional $(\mathrm{p}=0,957)$. En la figura 1 se muestra la distribución porcentual del tipo de cirugías realizada en cada cohorte.

En las complicaciones tempranas solo el cuadro febril fue más frecuente en el grupo con malla (4 pacientes). Se presentó un absceso de cúpula en una paciente con comorbilidad, que requirió drenaje quirúrgico, ninguna paciente requirió el retiro del material sintético. La infección urinaria fue más frecuente en el grupo de manejo tradicional (1 paciente), y en este grupo se presentó el único caso de incontinencia de fecales. No hubo diferencias en las otras variables, ni temprana ni tardía, en los dos grupos.

El porcentaje de las fallas y la comparación de la eficacia de las técnicas al año de seguimiento se observa en la tabla 2. Se encontraron diferencias estadísticamente significativas en el índice de recaídas del compartimiento anterior, tanto a los seis meses como al año de seguimiento, a favor de la corrección con mallas. 
Tabla 1. Distribución de las pacientes según técnica quirúrgica empleada, variables sociodemográficas

\section{y antecedentes quirúrgicos.}

\begin{tabular}{|c|c|c|c|}
\hline Variable & $\begin{array}{c}\text { Cohorte con } \\
\text { malla } \\
\mathbf{n}=31\end{array}$ & $\begin{array}{c}\text { Cohorte tratamiento } \\
\text { tradicional } \\
\mathbf{n}=30\end{array}$ & Valor de $\mathrm{p} *$ \\
\hline Edad & $61,65 \pm 10,89$ & $59,10 \pm 8,3$ & 0,30 \\
\hline Estrato 1, 2 y 3 & $51,6 \%(16)$ & $73,3 \%(22)$ & 0,08 \\
\hline Peso & $66,10 \pm 10,31$ & $62,50 \pm 8,94$ & 0,15 \\
\hline Tabaquismo & $16,1 \%(5)$ & $13,3 \%(4)$ & 0,95 \\
\hline Presencia de enfermedades del colágeno & $3,2 \%(1)$ & $3,3 \%(1)$ & - \\
\hline IMC $>=24$ & $54,8 \%(17)$ & $53,3 \%(16)$ & 0,91 \\
\hline Nulípara & $6,5 \%(2)$ & $6,7 \%(2)$ & 0,63 \\
\hline$>=3$ partos vaginales & $70,9 \%(22)$ & $73,3 \%(22)$ & 0,84 \\
\hline Partos por cesárea & $3,2 \%(1)$ & $6,7 \%(2)$ & 0,98 \\
\hline Histerectomía abdominal & $22,6 \%(7)$ & $3,3 \%(1)$ & 0,06 \\
\hline Histerectomía vaginal & $6,5 \%(2)$ & $0,0 \%(0)$ & - \\
\hline Corrección de cistorrectocele & $0,0 \%(0)$ & $0,0 \%(0)$ & - \\
\hline Fijación sacroespinosa & $0,0 \%(0)$ & $0,0 \%(0)$ & - \\
\hline Cistopexia & $12,9 \%(4)$ & $6,7 \%(2)$ & 0,70 \\
\hline Colposacropexia & $0,0 \%(0)$ & $0,0 \%(0)$ & - \\
\hline $\begin{array}{l}\text { Prolapso genital } \\
\text { Grado II } \\
\text { Grado III } \\
\text { Grado IV }\end{array}$ & $\begin{array}{c}9,7 \%(3) \\
80,6 \%(25) \\
9,7 \%(3)\end{array}$ & $\begin{array}{c}16,7 \%(5) \\
73,3 \%(22) \\
10,0 \%(3)\end{array}$ & $\begin{array}{l}0,67 \\
0,50 \\
0,70\end{array}$ \\
\hline
\end{tabular}

*Para la comparación de proporciones se utilizó el test de la $\mathrm{Chi}^{2}$ y para las variables continuas se utilizó el test de la T-Student.

Figura 1. Distribución de las pacientes según técnica quirúrgica empleada.

Cohorte con malla

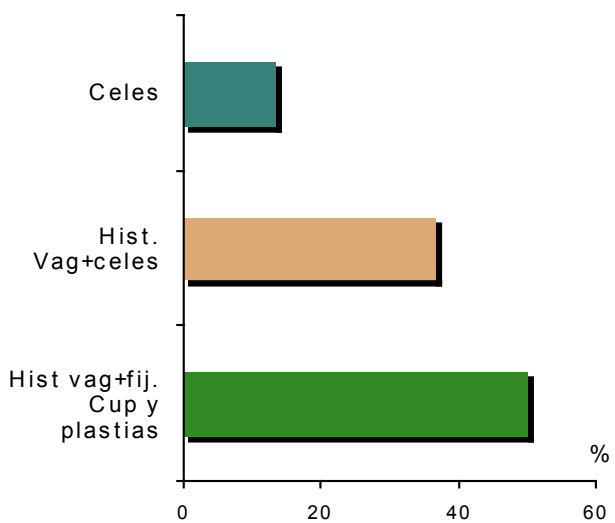

\section{Cohorte tradicional}

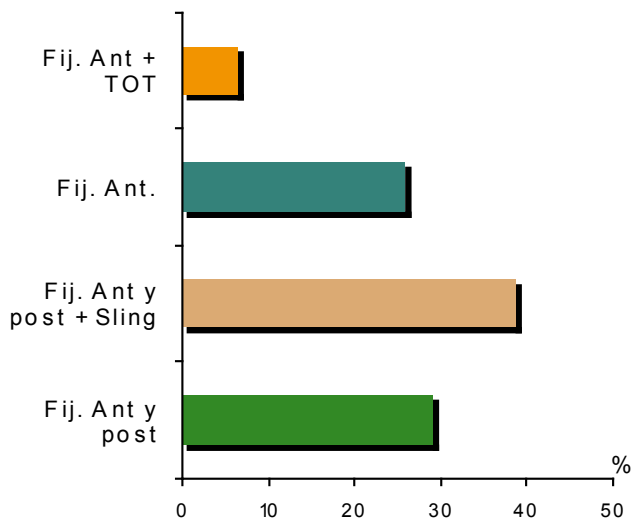

Fij. Ant. + TOT: fijación de cúpula plastia anterior y sling - Fij. Ant.: fijación de cúpula y plastia anterior - Fij. Ant. y post + Sling: fijación de cúpula plastia anterior y posterior y sling - Fij. Ant. y post: fijación de cúpula plastia anterior y posterior. - Celes: plastia anterior y posterior - Hist vag + celes: histerectomía vaginal y plastia anterior y posterior - Hist. vag + fij. Cup y plastias: histerectomía vaginal fijación de cúpula y plastia anterior y posterior. 


\begin{tabular}{|c|c|c|c|c|c|}
\hline Variable & $\begin{array}{c}\text { No. Px } \\
\text { Cohorte } \\
\text { intervenida } \\
\mathbf{n}=21\end{array}$ & $\begin{array}{c}\text { No. Px } \\
\text { Cohorte } \\
\text { tradicional } \\
\mathbf{n}=25\end{array}$ & $\mathbf{R R}$ & IC 95\% & Valor de $\mathrm{p}$ \\
\hline $\begin{array}{l}\text { Compartimiento anterior: } \\
\text { Grado II }\end{array}$ & $3(14,3 \%)$ & $10(40,0 \%)$ & 0,25 & $0,04-1,27$ & 0,05 \\
\hline Grado III & $1(4,8 \%)$ & $4(16,0 \%)$ & 0,26 & $0,01-2,92$ & 0,35 \\
\hline Grado IV & 0 & $1(4,0 \%)$ & $\mathrm{NC} * *$ & $\mathrm{NC} * *$ & $\mathrm{NC} * *$ \\
\hline $\begin{array}{l}\text { Total falla compartimiento } \\
\text { anterior }\end{array}$ & $4(19,1 \%)$ & $15(60,0 \%)$ & 0,32 & $0,12-0,81$ & 0,004 \\
\hline Falla compartimiento posterior & 0 & $3(12 \%)$ & $\mathrm{NC} * *$ & $\mathrm{NC} * *$ & $\mathrm{NC} * *$ \\
\hline Falla apical & $1(4,8 \%)$ & $3(12 \%)$ & 0,40 & $0,04-3,54$ & 0,73 \\
\hline
\end{tabular}

\section{DISCUSIÓN}

Este estudio encontró diferencias clínica y estadísticamente significativas al comparar las dos técnicas en cuanto a las recaídas del compartimiento anterior. La última revisión sistemática realizada por Cochrane en julio del $2007^{16}$ sobre el manejo quirúrgico del prolapso pélvico, tiene hallazgos que coinciden con lo encontrado en el seguimiento de las cohortes de esta investigación, privilegia la corrección del compartimiento anterior con material sintético y confirma la menor tasa de recaídas al usar esta técnica. Muestra ventajas la reparación única del prolapso apical por vía abdominal, pero no hay en esta revisión sistemática manejo de todas las distopias. La tasa de reintervenciones para prolapso apical no es significativa cuando compara la vía abdominal con la vaginal.

La vía de abordaje vaginal facilita al cirujano ginecológico la corrección de la mayoría de los trastornos de relajación del piso pélvico. La utilización de mallas tiene la ventaja de poder fijar la vagina de corta longitud, lo cual es una contraindicación en la fijación sacroespinosa clásica. La fijación a los sacroespinosos con mallas puede ser bilateral, logrando un ángulo vaginal normal y una amplitud adecuada de la cúpula, lo que representa menor dispareunia al compararse con otras técnicas quirúrgicas.
Otra ventaja adicional de la vía vaginal es el tratamiento simultáneo de la incontinencia urinaria durante el acto operatorio. No hay que olvidar que un porcentaje de pacientes con relajación severa del piso pélvico presentan incontinencia oculta durante su evaluación preoperatoria por obstrucción de la uretra, y lo ideal es que todas estas pacientes tengan un estudio urodinámico previo a la cirugía, advirtiendo que se realice con reducción del prolapso para detectar la incontinencia. ${ }^{17}$

En el compartimiento anterior es donde se debe concentrar el máximo esfuerzo de la reparación, porque este sitio puede presentar diferentes tipos de fallas, que se manifiestan clínicamente como "cistoceles" centrales, fallas o desinserciones laterales o paravaginales de la vagina a la fascia del músculo obturador y específicamente del arco tendíneo, que se extiende desde el pubis hasta la espina ciática y posteriores, cuando cerca al anillo pericervical se puede desinsertar la fascia, y que ameritan reparaciones específicas para su control; el uso de material sintético ha permitido el control simultáneo de estos defectos.

Debilidades del estudio: el seguimiento es corto aun para poder evaluar los resultados a mediano y largo plazo.

Fortalezas: evaluación enmascarada a los seis meses y el año. 


\section{CONCLUSIONES}

El manejo con mallas en la cirugía de reparación del piso pélvico parece ser una alternativa promisoria en la prevención de la recurrencia o recaída del compartimiento anterior comparada con el manejo tradicional. La morbilidad de ambas técnicas es comparable. Estos hallazgos deberán ser confirmados mediante ensayos clínicos controlados.

\section{REFERENCIAS}

1. Luber KM, Boero S, Choe JY. The demographics of pelvic floor disorders: current observations and future projections. Am J Obstet Gynecol 2001;184:1496503.

2. Echavarría LG. Fijación sacroespinosa en el manejo del prolapso de cúpula vaginal y hernia pélvica: diez años de experiencia (1992-2002). Rev Colomb Obstet Ginecol 2004;55:30-4.

3. Cruikshank S, Muniz M. Outcomes study: A comparison of cure rate in 695 patients undergoing sacrospinous ligament fixation alone and with other site specific procedures-- a 16 year study. Am J Obstet Gynecol 2003;188:1509-12.

4. Braun H, Rojas I, González F, Fernández M, Ortiz JA. Prolapso genital severo: consideraciones clínicas, fisiopatológicas y de técnica quirúrgica al momento de su corrección. Rev Chil Obstet Ginecol 2004;69:149-56.

5. Wong MY, Harmanli OH, Agar M, Dandolu V, Grody T. Collagen content of nonsupport tissue in pelvic organ prolapse and stress urinary incontinence. Am J Obstet Gynecol 2003;189:1597-9.

6. Smith PH, Heimer G, Norgren A, Ulmsten U. Localization of steroid hormone receptors in the pelvic muscles. Eur J Obstet Gynecol Reprod Biol 1993;50:83-5.
7. Carter JE. Enterocele repair and vaginal vault suspension. Curr Opin Obstet Gynecol 2000;12: 321-30.

8. Bump RC, Norton PA. Epidemiology and natural history of pelvic floor dysfunction. Obstet Gynecol Clin North Am 1998;25:723-46.

9. Goh JT. Biomechanical and biochemical assessments for pelvic organ prolapse. Curr Opin Obstet Gynecol 2003;15:391-4.

10. Maher CF, Qatawneh AM, Dwyer PL, Carey MP, Cornish A, Schluter PJ. Abdominal sacral colpopexy or vaginal sacrospinous colpopexy for vaginal vault prolapse: A prospective randomized study. Am J Obstet Gynecol 2004;190:20-6.

11. Carey MP, Dwyer PL. Genital prolapse: vaginal versus abdominal route of repair. Curr Opin Obstet Gynecol 2001;13:499-505.

12. Silva WA, Karram MM. Scientific basis for use of grafts during vaginal reconstructive procedures. Curr Opin Obstet Gynecol 2005;17:519-29.

13. Griffis K, Hale DS. Grafts in pelvic reconstructive surgery. Clin Obstet Gynecol 2005;48:713-23.

14. DeLancey JO. Childbirth, continence and the pelvic floor. N Engl J Med 1993;329:1956-7.

15. Cervigni M, Natale F. The use of synthetics in the treatment of pelvic organ prolapse. Curr Opin Urol 2001;11:429-35.

16. Maher C, Baessler K, Glazener CM, Adams EJ, Hagen S. Surgical management of pelvic organ prolapse in women. Cochrane Database Syst Rev 2007;(3): CD004014.

17. Veronikis DK, Nichols DH, Wakamatsu MM. The incidence of low-pressure urethra as a function of prolapse reducing technique in patients with massive pelvic organ prolapse (maximun descent at all vaginal sites). Am J Obstet Gynecol 1997;177:1305-13. 